\title{
Fuzzy Retractions of Fuzzy Open Flat Robertson-Walker Space
}

\author{
A. E. El-Ahmady ${ }^{1,2}$ and A. S. Al-Luhaybi ${ }^{1}$ \\ ${ }^{1}$ Mathematics Department, Faculty of Science, Taibah University, Madinah, Saudi Arabia \\ ${ }^{2}$ Mathematics Department, Faculty of Science, Tanta University, Tanta 31527, Egypt
}

Correspondence should be addressed to A. E. El-Ahmady; a_elahmady@hotmail.com

Received 24 April 2012; Accepted 28 August 2012

Academic Editor: Ching-Hung Lee

Copyright (c) 2013 A. E. El-Ahmady and A. S. Al-Luhaybi. This is an open access article distributed under the Creative Commons Attribution License, which permits unrestricted use, distribution, and reproduction in any medium, provided the original work is properly cited.

\begin{abstract}
Our aim in the present paper is to introduce and study new types of fuzzy retractions of fuzzy open flat Robertson-Walker $\widetilde{W}^{4}$ model. New types of the fuzzy deformation retracts of $\widetilde{W}^{4}$ model are obtained. The relations between the fuzzy foldings and the fuzzy deformation retracts of $\widetilde{W}^{4}$ model are deduced. Types of fuzzy minimal retractions are also presented. New types of homotopy maps are deduced. New types of conditional fuzzy folding are presented. Some commutative diagrams are obtained.
\end{abstract}

\section{Introduction and Background}

Robertson-Walker space represents one of the most intriguing and emblematic discoveries in the history of geometry. Although if it were introduced for a purely geometrical purpose, they came into prominence in many branches of mathematics and physics. This association with applied science and geometry generated synergistic effect: applied science gave relevance to Robertson-Walker space and RobertsonWalker space allowed formalizing practical problems [1-6]. As is well known, the theory of retractions is always one of interesting topics in Euclidian and Non-Euclidian spaces and it has been investigated from the various viewpoints by many branches of topology and differential geometry [7-11]. There are many diverse applications of certain phenomena for which it is impossible to get relevant data. It may not be possible to measure essential parameters of a process such as the temperature inside molten glass or the homogeneity of a mixture inside some tanks. The required measurement scale may not exist at all, such as in the case of evaluation of offensive smells, evaluating the taste of foods or medical diagnoses by touching $[7,8,12-18]$. The aim of the present paper is to describe the above phenomena geometrically, specifically concerned with the study of the new types of fuzzy retractions, fuzzy deformation retracts, and fuzzy folding of fuzzy open flat Robertson-Walker $\widetilde{W}^{4}$ model. A fuzzy manifold is manifold which has a physical character. This character is represented by the density function $\mu$, where $\mu \in[0,1][7,8,12]$.

A fuzzy subset $(\underline{A}, \mu)$ of a fuzzy manifold $(\underline{M}, \mu)$ is called a fuzzy retraction of $(\widetilde{M}, \mu)$ if there exist a continuous map $\widetilde{r}$ : $(\underline{M}, \mu) \rightarrow(\underline{A}, \mu)$ such that $\tilde{r}(a, \mu(a))=(a, \mu(a))$, for all $a \in$ A, $\mu \in[0,1][7,8,12]$.

A fuzzy subset $(\bar{M}, \widetilde{\mu})$ of a fuzzy manifold $(\underline{M}, \mu)$ is called a fuzzy deformation retract if there exists a fuzzy retraction $\tilde{r}:(\underline{M}, \mu) \rightarrow(\underline{M}, \tilde{\mu})$ and a fuzzy homotopy $\widetilde{F}:$ $(\underline{M}, \mu) \times I \rightarrow(\underline{M}, \mu)[7,13,14]$ such that

$$
\begin{gathered}
\tilde{F}((x, \mu), 0)=(x, \mu), \quad x \in M, \\
\widetilde{F}((x, \mu), 1)=\widetilde{r}(x, \mu),
\end{gathered}
$$

$\widetilde{F}((a, \mu), t)=(a, \mu)$, for all $(a, \mu) \in \bar{M}, t \in I, \mu \in[0,1]$, where $\widetilde{r}(x, \mu)$ is the retraction mentioned above.

Topological folding of fuzzy open flat Robertson-Walker space $\widetilde{W}^{4}$ model $[7,8]$. A map $\widetilde{\mathfrak{F}}: \widetilde{W}^{4} \rightarrow \widetilde{W}^{4}$ is said to be an isometric folding of $\widetilde{W}^{4}$ model into itself if and only if for any piecewise fuzzy geodesic path $\gamma: J \rightarrow \widetilde{W}^{4}$ the induced path $\widetilde{F} \circ \gamma: J \rightarrow \widetilde{W}^{4}$ is a piecewise fuzzy geodesic and of the same length as $\gamma$, where $J=[0,1]$. If $\widetilde{\mathfrak{F}}$ does not preserve lengths, then $\widetilde{\mathfrak{F}}$ is a topological folding of fuzzy RobertsonWalker space $\widetilde{W}^{4}$ model [12-14].

The fuzzy folding of $\bigcup \widetilde{M}_{i} \subseteq \widetilde{W}^{4}$ model is a folding $\tilde{f}$ : $\bigcup \widetilde{M}_{i} \rightarrow \bigcup \widetilde{M}_{i}$ such that $\widetilde{f}(\widetilde{M})=\widetilde{M}$ and any $\widetilde{M}_{i}$ belong to 


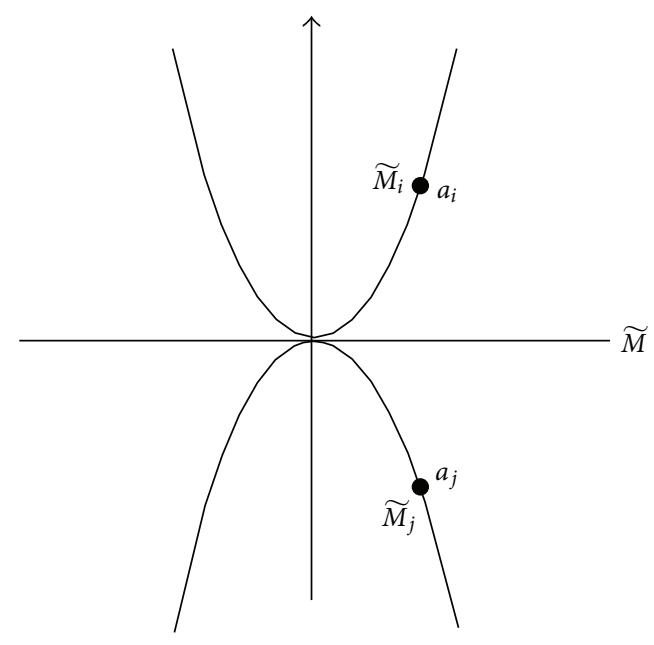

FIGURE 1

the upper hypermanifolds $\exists \widetilde{M}_{j}$ down $\widetilde{M}$ such that $\mu_{i}=\mu_{j}$ for every corresponding points, that is, $\mu\left(a_{i}\right)=\mu\left(a_{j}\right)$ [15]. See Figure 1.

\section{Main Results}

Theorem 1. The fuzzy retractions of $\widetilde{W}^{4}$ model are the fuzzy unit hyperboloid, fuzzy hyperbolic, fuzzy hypersphere, fuzzy circle, and fuzzy minimal manifolds.

Proof. Consider the $\widetilde{W}^{4}$ model with metric

$$
\begin{aligned}
d s^{2}(\mu)= & d \chi^{2}(\mu)+\sinh ^{2} \chi(\mu) \\
& \times\left(d \theta^{2}(\mu)+\sin ^{2} \theta(\mu) d \emptyset^{2}(\mu)\right), \quad \mu \in[0,1] .
\end{aligned}
$$

The coordinate of $\widetilde{W}^{4}$ model is

$$
\begin{aligned}
& \tilde{x}_{1}=\sinh \chi(\mu) \sin \theta(\mu) \cos \emptyset(\mu), \\
& \tilde{x}_{2}=\sinh \chi(\mu) \sin \theta(\mu) \sin \emptyset(\mu), \\
& \tilde{x}_{3}=\sinh \chi(\mu) \cos \theta(\mu), \\
& \tilde{x}_{4}=\cosh \chi(\mu),
\end{aligned}
$$

where the ranges are $0 \leq \chi(\mu)<\infty, 0 \leq \theta(\mu)<\pi$, and $0 \leq \emptyset(\mu)<2 \pi$.

Now, we use Lagrangian equations:

$$
\frac{d}{d s}\left(\frac{\partial T}{\partial \psi^{\prime}(\mu)_{i}}\right)-\frac{\partial T}{\partial \psi(\mu)_{i}}=0, \quad i=1,2,3,4 .
$$

To find a fuzzy geodesic which is a fuzzy subset of $\widetilde{W}^{4}$ model, since

$$
T=\frac{1}{2}\left\{\grave{\chi}^{2}(\mu)+\sinh ^{2} \chi(\mu)\left(\grave{\theta}^{2}(\mu)+\sin ^{2} \theta(\mu) \grave{\emptyset}^{2}(\mu)\right)\right\},
$$

then the Lagrangian equations for $\widetilde{W}^{4}$ model are

$$
\begin{aligned}
& \frac{d}{d s}(\grave{\chi}(\mu))-(\sinh \chi(\mu) \cosh \chi(\mu) \\
& \left.\times\left(\grave{\theta}^{2}(\mu)+\sin ^{2} \theta(\mu) \grave{\emptyset}^{2}(\mu)\right)\right)=0, \\
& \frac{d}{d s}\left(\sinh ^{2} \chi(\mu) \grave{\theta}(\mu)\right) \\
& -\left(\sinh ^{2} \chi(\mu) \sin \theta(\mu) \cos \theta(\mu) \grave{\emptyset}^{2}(\mu)\right)=0, \\
& \frac{d}{d s}\left(\sin ^{2} \chi(\mu) \sin ^{2} \theta(\mu) \grave{\emptyset}(\mu)\right)=0, \\
& -(\grave{\chi}(\mu))+\left(\sinh \chi(\mu) \cosh \chi(\mu) \grave{\theta}^{2}(\mu)\right. \\
& \left.+\sinh ^{2} \chi(\mu) \grave{\theta}(\mu)\right) \\
& +\left(\sinh \chi(\mu) \cosh \chi(\mu) \sin ^{2} \theta(\mu) \grave{\emptyset}^{2}(\mu)\right. \\
& +\sinh ^{2} \chi(\mu) \sin \theta(\mu) \cos \theta(\mu) \grave{\emptyset}^{2}(\mu) \\
& \left.+\sinh ^{2} \chi(\mu) \sin ^{2} \theta(\mu) \emptyset(\grave{\mu})\right)=0 .
\end{aligned}
$$

From (7) we obtain $\sinh ^{2} \chi(\mu) \sin ^{2} \theta(\mu) \grave{\emptyset}(\mu)=$ constant, say $\beta_{1}$, if $\beta_{1}=0$, we obtain the following cases.

If initially $\theta$ equal $\pi / 6$ or $\pi / 4$ and $\pi / 3$ hence we obtain the following fuzzy geodesics of fuzzy unit hyperboloid $\widetilde{H}_{1}^{3}, \widetilde{H}_{2}^{3}$, and $\widetilde{H}_{3}^{3}$, respectively. Also, if $\theta=\pi / 2$, hence we obtain the following coordinates of $\widetilde{W}^{4}$ model given by

$$
\begin{gathered}
\tilde{x}_{1}=\sinh \chi(\mu) \cos \emptyset(\mu), \\
\tilde{x}_{2}=\sinh \chi(\mu) \sin \emptyset(\mu), \\
\tilde{x}_{3}=0, \\
\tilde{x}_{4}=\cosh \chi(\mu),
\end{gathered}
$$

which is a fuzzy hyperbolic $\widetilde{H}_{1}^{2},-\widetilde{x}_{4}^{2}+\widetilde{x}_{1}^{2}+\widetilde{x}_{2}^{2}+\widetilde{x}_{3}^{2}=-1$, which is a fuzzy geodesic retraction. Now, If $\emptyset=\pi / 6$ or $\pi / 4$ and $\pi / 3$ hence we get the fuzzy unit hyperboloid retractions $\widetilde{H}_{4}^{3}, \widetilde{H}_{5}^{3}$ and, $\widetilde{H}_{6}^{3}$ in $\widetilde{W}^{4}$ model, respectively. Also, in a special case if $\emptyset=\pi / 2$ or $\pi$ and $3 \pi / 2$ hence we get the fuzzy hyperbolic geodesics $\widetilde{H}_{2}^{2}, \widetilde{H}_{3}^{2}$, and $\widetilde{H}_{4}^{2}$ in $\widetilde{W}^{4}$ model, respectively. In a special case if $\chi=\pi / 2$, hence we get the coordinates of $\widetilde{W}^{4}$ model represented by

$$
\begin{gathered}
\tilde{x}_{1}=\sin \theta(\mu) \cos \emptyset(\mu), \\
\tilde{x}_{2}=\sin \theta(\mu) \sin \emptyset(\mu), \\
\tilde{x}_{3}=\cos \theta(\mu) \\
\tilde{x}_{4}=0,
\end{gathered}
$$

which is a fuzzy sphere $\widetilde{S}_{1}^{2},-\widetilde{x}_{4}^{2}+\tilde{x}_{1}^{2}+\tilde{x}_{2}^{2}+\widetilde{x}_{3}^{2}=-1$, which 
is a fuzzy geodesic retraction. Also, If $\emptyset=90$, and $\chi=90$ we obtain the fuzzy retraction, $\widetilde{S}^{1}=(0, \sin \theta(\mu), \cos \theta(\mu), 0)$, which is a fuzzy circle $\widetilde{S}^{1}$. Again, If $\chi=\pi$, we get the following minimal fuzzy geodesic $\widetilde{W}^{0}(0,0,0,1)$ in $\widetilde{W}^{4}$ model.

In what follows, we present some cases of fuzzy deformation retracts of $\widetilde{W}^{4}$ model. The fuzzy deformation retract of $\widetilde{W}^{4}$ model is $\eta: \widetilde{W}^{4} \times I \rightarrow \widetilde{W}^{4}$, where $I$ is the closed interval $[0,1]$, present as

$$
\begin{aligned}
& \tilde{\eta}(x, h):\{\sinh \chi(\mu) \sin \theta(\mu) \cos \emptyset(\mu), \\
& \sinh \chi(\mu) \sin \theta(\mu) \sin \emptyset(\mu), \\
&\sinh \chi(\mu) \cos \theta(\mu), \cosh \chi(\mu)\} \times I \\
& \longrightarrow\{\sinh \chi(\mu) \sin \theta(\mu) \cos \emptyset(\mu), \\
& \sinh \chi(\mu) \sin \theta(\mu) \sin \emptyset(\mu), \\
&\sinh \chi(\mu) \cos \theta(\mu), \cosh \chi(\mu)\} .
\end{aligned}
$$

The fuzzy deformation retract of $\widetilde{W}^{4}$ model into the fuzzy minimal geodesic $\widetilde{W}^{0}$ is

$$
\begin{aligned}
\widetilde{\eta}(m, h)=(1+h)\{ & \sinh \chi(\mu) \sin \theta(\mu) \cos \emptyset(\mu), \\
& \sinh \chi(\mu) \sin \theta(\mu) \sin \emptyset(\mu), \\
& \sinh \chi(\mu) \cos \theta(\mu), \cosh \chi(\mu)\} \\
+ & \tan \frac{\pi h}{4}\{0,0,0,1\},
\end{aligned}
$$

where

$$
\begin{aligned}
& \widetilde{\eta}(m, 0)=\{\sinh \chi(\mu) \sin \theta(\mu) \cos \emptyset(\mu), \\
& \sinh \chi(\mu) \sin \theta(\mu) \sin \emptyset(\mu), \sinh \chi(\mu) \cos \theta(\mu), \\
&\cosh \chi(\mu)\}, \\
& \tilde{\eta}(m, 1)=\{0,0,0,1\} .
\end{aligned}
$$

The fuzzy deformation retract of $\widetilde{W}^{4}$ model into the fuzzy hyperboloid $\widetilde{H}_{1}^{2}$ is

$$
\begin{gathered}
\widetilde{\eta}(m, h)=\cos \frac{\pi h}{2}\{\sinh \chi(\mu) \sin \theta(\mu) \cos \emptyset(\mu), \\
\sinh \chi(\mu) \sin \theta(\mu) \sin \emptyset(\mu), \\
\sinh \chi(\mu) \cos \theta(\mu), \cosh \chi(\mu)\} \\
+\sin \frac{\pi h}{2}\{\sinh \chi(\mu) \cos \emptyset(\mu), \sinh \chi(\mu) \\
\times \sin \emptyset(\mu), 0, \cosh \chi(\mu)\}
\end{gathered}
$$

Now, we are going to discuss the fuzzy folding $\widetilde{\widetilde{F}}$ of $\widetilde{W}^{4}$ model. Let $\widetilde{\mathfrak{F}}: \widetilde{W}^{4} \rightarrow \widetilde{W}^{4}$, where

$$
\widetilde{\mathfrak{F}}\left(\widetilde{x}_{1}, \tilde{x}_{2}, \tilde{x}_{3}, \tilde{x}_{4}\right)=\left(\tilde{x}_{1}, \tilde{x}_{2},\left|\tilde{x}_{3}\right|, \tilde{x}_{4}\right) .
$$

An isometric fuzzy folding of $\widetilde{W}^{4}$ model into itself may be defined by

$$
\begin{aligned}
& \widetilde{\widetilde{F}}:\{\sinh \chi(\mu) \sin \theta(\mu) \cos \emptyset(\mu), \sinh \chi(\mu) \sin \theta(\mu) \\
& \times\sin \emptyset(\mu), \sinh \chi(\mu) \cos \theta(\mu), \cosh \chi(\mu)\} \\
& \longrightarrow\{\sinh \chi(\mu) \sin \theta(\mu) \cos \emptyset(\mu), \sinh \chi(\mu) \sin \theta(\mu) \\
&\times \sin \emptyset(\mu),|\sinh \chi(\mu) \cos \theta(\mu)|, \cosh \chi(\mu)\} .
\end{aligned}
$$

The fuzzy deformation retract of the fuzzy folded $\widetilde{\mathfrak{F}}\left(\widetilde{W}^{4}\right)$ model into the fuzzy folded geodesic $\widetilde{\mathfrak{F}}\left(\widetilde{W}^{0}\right)$ is

$$
\begin{aligned}
& \widetilde{\eta}_{\widetilde{F}}:\{\sinh \chi(\mu) \sin \theta(\mu) \cos \emptyset(\mu), \sinh \chi(\mu) \sin \theta(\mu) \\
&\sin \emptyset(\mu),|\sinh \chi(\mu) \cos \theta(\mu)|, \cosh \chi(\mu)\} \times I \\
& \longrightarrow\{\sinh \chi(\mu) \sin \theta(\mu) \cos \emptyset(\mu), \sinh \chi(\mu) \\
& \times \sin \theta(\mu) \sin \emptyset(\mu),|\sinh \chi(\mu) \cos \theta(\mu)|, \\
&\cosh \chi(\mu)\} .
\end{aligned}
$$

with

$$
\begin{aligned}
\tilde{\eta}_{\widetilde{F}}(m, h)=(1+h)\{ & \sinh \chi(\mu) \sin \theta(\mu) \cos \emptyset(\mu), \\
& \sinh \chi(\mu) \sin \theta(\mu) \sin \emptyset(\mu), \\
& |\sinh \chi(\mu) \cos \theta(\mu)|, \cosh \chi(\mu)\} \\
+ & \tan \frac{\pi h}{4}\{0,0,0,1\} .
\end{aligned}
$$

The fuzzy deformation retract of the fuzzy folded $\widetilde{\mathfrak{F}}\left(\widetilde{W}^{4}\right)$ model into the fuzzy folded geodesic $\widetilde{\mathfrak{F}}\left(\widetilde{H}_{1}^{2}\right)$ is

$$
\begin{aligned}
\widetilde{\eta}_{\widetilde{F}}(m, h)=\cos \frac{\pi h}{2}\{\sinh \chi(\mu) \sin \theta(\mu) \cos \emptyset(\mu), \\
\sinh \chi(\mu) \sin \theta(\mu) \sin \emptyset(\mu), \\
|\sinh \chi(\mu) \cos \theta(\mu)|, \cosh \chi(\mu)\} \\
+\sin \frac{\pi h}{2}\{\sinh \chi(\mu) \cos \emptyset(\mu), \\
\\
\sinh \chi(\mu) \sin \emptyset(\mu), 0, \cosh \chi(\mu)\} .
\end{aligned}
$$

Then, the following theorem has been proved. 
Theorem 2. Under the defined fuzzy folding and any fuzzy folding homeomorphic to this type of fuzzy folding, the fuzzy deformation retract of the fuzzy folded $\widetilde{\mathfrak{F}}\left(\widetilde{W}^{4}\right)$ model into the fuzzy folded geodesics is the same as the fuzzy deformation retract of $\widetilde{W}^{4}$ model into the fuzzy geodesics.

Proof. Now, let the fuzzy folding be defined by $\widetilde{\widetilde{F}}^{*}: \widetilde{W}^{4} \rightarrow$ $\widetilde{W}^{4}$, where

$$
\widetilde{\mathfrak{F}}^{*}\left(\widetilde{x}_{1}, \widetilde{x}_{2}, \widetilde{x}_{3}, \widetilde{x}_{4}\right)=\left(\left|\widetilde{x}_{1}\right|, \widetilde{x}_{2}, \widetilde{x}_{3}, \widetilde{x}_{4}\right) .
$$

The isometric fuzzy folded $\widetilde{\mathfrak{F}}^{*}\left(\widetilde{W}^{4}\right)$ model is

$$
\begin{aligned}
& \overline{\widetilde{R}}=\{|\sinh \chi(\mu) \sin \theta(\mu) \cos \emptyset(\mu)|, \\
& \sinh \chi(\mu) \sin \theta(\mu) \sin \emptyset(\mu), \\
&\sinh \chi(\mu) \cos \theta(\mu), \cosh \chi(\mu)\} .
\end{aligned}
$$

The fuzzy deformation retract of the fuzzy folded $\widetilde{\mho}^{*}\left(\widetilde{W}^{4}\right)$ model into the fuzzy folded geodesic $\widetilde{\mathfrak{F}}^{*}\left(\widetilde{S}_{1}^{2}\right)$ is

$$
\begin{aligned}
& \widetilde{\eta_{\mathfrak{F}^{*}}}(m, h)=(1-h) \\
& \times\{|\sinh \chi(\mu) \sin \theta(\mu) \cos \emptyset(\mu)|, \\
& \sinh \chi(\mu) \sin \theta(\mu) \sin \emptyset(\mu), \\
&\sinh \chi(\mu) \cos \theta(\mu), \cosh \chi(\mu)\} \\
&+ \tan \frac{\pi \mathrm{h}}{4}\{|\sin \theta(\mu) \cos \emptyset(\mu)|, \\
&\sin \theta(\mu) \sin \emptyset(\mu), \cos \theta(\mu), 0\} .
\end{aligned}
$$

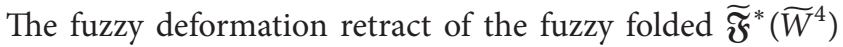
model into the fuzzy folded geodesic $\widetilde{\widetilde{F}}^{*}\left(\widetilde{H}_{3}^{2}\right)$ is

$$
\begin{aligned}
& \widetilde{\eta_{\mathfrak{F}^{*}}}(m, h)=\cos \frac{\pi \mathrm{h}}{2}\{\mid \sinh \chi(\mu) \sin \theta(\mu) \cos \emptyset(\mu) \mid, \\
& \sinh \chi(\mu) \sin \theta(\mu) \sin \emptyset(\mu), \\
&\sinh \chi(\mu) \cos \theta(\mu), \cosh \chi(\mu)\} \\
&+\sin \frac{\pi \mathrm{h}}{2}\{|\sinh \chi(\mu) \sin \theta(\mu)|, 0, \\
&\sinh \chi(\mu) \cos \theta(\mu), \cosh \chi(\mu)\} .
\end{aligned}
$$

Then, the following theorem has been proved.

Theorem 3. Under the defined fuzzy folding and any fuzzy folding homeomorphic to this type of fuzzy folding, the fuzzy deformation retract of the fuzzy folded $\widetilde{F}^{*}\left(\widetilde{W}^{4}\right)$ model into the fuzzy folded geodesics is different from the fuzzy deformation retract of $\widetilde{W}^{4}$ model into the fuzzy geodesics.
Theorem 4. Let $\widetilde{H}^{3} \subset \widetilde{W}^{4}$ be a fuzzy hyperboloid in $\widetilde{W}^{4}$ model which is homeomorphic to $\left\{\widetilde{D}^{2}-\widetilde{\beta}\right\} \subset \widetilde{R}^{3}$, and $\widetilde{r}_{1}: \widetilde{H}^{3} \rightarrow \widetilde{H}^{2} a$ fuzzy retraction. Then, there is an induced fuzzy retraction $\widetilde{r}_{2}$ : $\left\{\widetilde{D}^{2}-\widetilde{\beta}\right\} \rightarrow \widetilde{D}^{1}$ such that the following diagram is commutative:

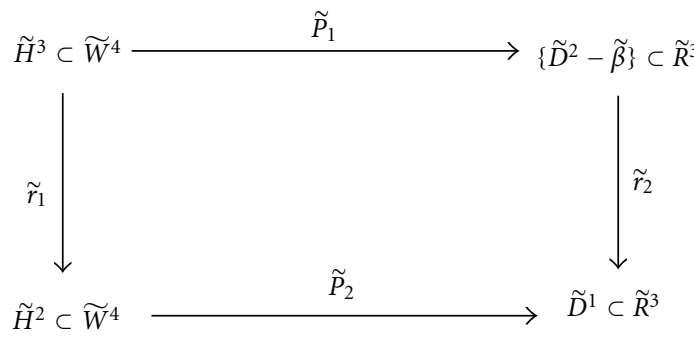

Proof. Under the condition $\theta=\pi / 2$, then $\widetilde{r}_{1}: \widetilde{H}^{3} \rightarrow \widetilde{H}^{2}$ is defined as $\widetilde{r}_{1}\{\sinh \chi(\mu) \sin \theta(\mu) \cos \emptyset(\mu), \sinh \chi(\mu) \sin \theta(\mu)$ $\sin \emptyset(\mu), \sinh \chi(\mu) \cos \theta(\mu), \cosh \chi(\mu)\}=\{\sinh \chi(\mu) \cos \emptyset(\mu)$, $\sinh \chi(\mu) \sin \emptyset(\mu), 0, \cosh \chi(\mu)\}$ also under the condition $\theta=$ $\pi / 2$, then $\widetilde{r}_{2}:\left\{\widetilde{D}^{2}-\widetilde{\beta}\right\} \rightarrow \widetilde{D}^{1}$ is defined as $\widetilde{r}_{2}\{\{\sin \theta(\mu)$ $\cos \emptyset(\mu), \sin \theta(\mu) \sin \emptyset(\mu), \cos \theta(\mu), 0\}-\widetilde{\beta}\}=\{\cos \emptyset(\mu)$, $\sin \emptyset(\mu), 0,0\}$. Under the homeomorphism map $\widetilde{P}_{1}: \widetilde{H}^{3} \subset$ $\widetilde{W}^{4} \rightarrow\left\{\widetilde{D}^{2}-\widetilde{\beta}\right\} \subset \widetilde{R}^{3}$ and $\widetilde{P}_{2}: \widetilde{H}^{2} \subset \widetilde{W}^{4} \rightarrow \widetilde{D}^{1} \subset \widetilde{R}^{3}$. This proves that the diagram is commutative.

Also, the corresponding relations are described as that is,

$$
\overline{\widetilde{r}_{2}} \circ \overline{\widetilde{p}_{1}}=\overline{\overline{p_{2}}} \circ \overline{\overline{r_{1}}}, \quad \underline{\widetilde{r_{2}}} . \overline{\widetilde{p_{1}}}=\underline{\widetilde{p_{2}}} \circ \underline{\widetilde{r_{1}}} .
$$

Theorem 5. Let $\widetilde{H}^{3} \subset \widetilde{W}^{4}$ be a fuzzy hyperboloid which is homeomorphic to $\left\{\widetilde{D}^{2}-\widetilde{\beta}\right\} \subset \widetilde{R}^{3}$, and $\lim _{n \rightarrow \infty} \widetilde{r}_{n}: \widetilde{H}^{3} \rightarrow \widetilde{H}^{2}$ a limit fuzzy retraction. Then, there is an induced limit fuzzy retraction $\lim _{n \rightarrow \infty} \widetilde{r}_{n+1}:\left\{\widetilde{D}^{2}-\widetilde{\beta}\right\} \rightarrow \widetilde{D}^{1}$ such that the following diagram is commutative:

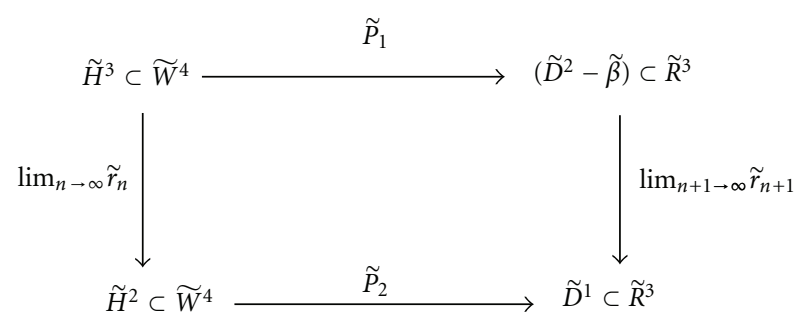

Proof. Since $\lim _{n \rightarrow \infty} \widetilde{r}_{n}: \widetilde{H}^{3} \rightarrow \widetilde{H}^{2}$ and $\lim _{n+1 \rightarrow \infty} \widetilde{r}_{n+1}$ : $\left\{\widetilde{D}^{2}-\widetilde{\beta}\right\} \rightarrow \widetilde{D}^{1}$, under the homeomorphism map $\widetilde{P}_{1}: \widetilde{H}^{3}$ $\subset \widetilde{W}^{4} \rightarrow\left\{\widetilde{D}^{2}-\widetilde{\beta}\right\} \subset \widetilde{R}^{3}$ and $\widetilde{P}_{2}: \widetilde{H}^{2} \subset \widetilde{W}^{4} \rightarrow \widetilde{D}^{1} \subset$ $\widetilde{R}^{3}$. This proves that the diagram is commutative. Also, the corresponding relations are presented as

$$
\begin{aligned}
& \overline{\lim _{n+1 \rightarrow \infty} r_{n+1}} \circ \overline{\widetilde{p}_{1}}=\overline{\widetilde{p}_{2}} \circ \overline{\widetilde{\lim }_{n \rightarrow \infty} r_{n}}, \\
& \varlimsup_{n+1 \rightarrow \infty} r_{n+1} \circ \underline{\tilde{p}_{1}}=\underline{\tilde{p}_{2}} \circ \varlimsup_{n \rightarrow \infty} r_{n} .
\end{aligned}
$$


Theorem 6. If the fuzzy deformation retract of the fuzzy hyperboloid $\widetilde{H}^{3} \subset \widetilde{W}^{4}$ is $\widetilde{D}: \widetilde{H}^{3} \times I \rightarrow \widetilde{H}^{3}$, the fuzzy retraction of $\widetilde{H}^{3} \subset \widetilde{W}^{4}$ is $\widetilde{r}: \widetilde{H}^{3} \rightarrow \widetilde{H}^{2}$, and the limit of the fuzzy folding of $\widetilde{H}^{3}$ is $\lim _{m \rightarrow \infty} \widetilde{f}_{m}: \widetilde{H}^{3} \rightarrow \widetilde{H}^{2}$. Then there are induced fuzzy deformation retract, fuzzy retraction, and the limit of the fuzzy foldings such that the following diagram is commutative.

Proof. Let the fuzzy deformation retract of $\widetilde{H}^{3} \subset \widetilde{W}^{4}$ be $\widetilde{D}_{1}$ : $\widetilde{H}^{3} \times I \rightarrow \widetilde{H}^{3}$; the fuzzy retraction of $\widetilde{H}^{3} \times I$ is defined by $\widetilde{r_{1}}:\left(\widetilde{H}^{3} \times I\right) \rightarrow \widetilde{H}^{2} \times I, \lim _{m \rightarrow \infty} \widetilde{f}_{m}: \widetilde{D}_{1}\left(\widetilde{H}^{3} \times I\right) \rightarrow$ $\widetilde{H}^{2}$, the fuzzy deformation retract of $\widetilde{r}_{1}\left(\widetilde{H}^{3} \times I\right)$ is $\widetilde{D}_{2}$ : $\widetilde{r_{1}}\left(\widetilde{H}^{3} \times I\right) \rightarrow \widetilde{H}^{2}$, the fuzzy retraction of $\lim _{m \rightarrow \infty} \widetilde{f}_{m}$. $\left(\widetilde{D}_{1}\left(\widetilde{H}^{3} \times I\right)\right)$ is given by $\widetilde{r_{2}}: \lim _{m \rightarrow \infty} \widetilde{f}_{m}\left(\widetilde{D}_{1}\left(\widetilde{H}^{3} \times I\right)\right) \rightarrow$ $\widetilde{H}^{1}$, and $\lim _{m+1 \rightarrow \infty} \widetilde{f}_{m+1}: \widetilde{D}_{2}\left(\widetilde{r}_{1}\left(\widetilde{H}^{3} \times I\right)\right) \rightarrow \widetilde{H}^{1}, \widetilde{H}^{1}$ is a 1 -dimensional space. Hence, the following diagram is commutative,

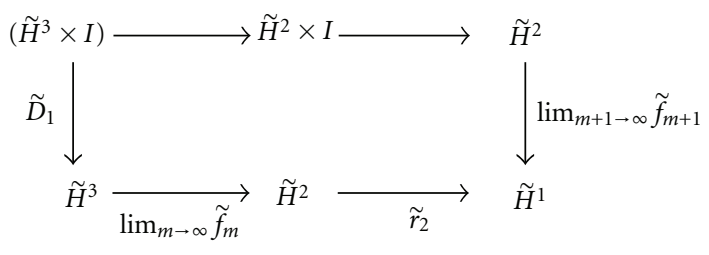

that is, $\lim _{m+1 \rightarrow \infty} \widetilde{f}_{m+1} \circ \widetilde{D}_{2} \circ \widetilde{r}_{1}\left(\widetilde{H}^{3} \times I\right)=\widetilde{r}_{2} \circ \lim _{m \rightarrow \infty} \widetilde{f}_{m}$ 。 $\widetilde{D_{1}}$.

Theorem 7. Let $\widetilde{H}^{3} \subset \widetilde{W}^{4}$ be the fuzzy hyperboloid, then the relation between the fuzzy folding $\widetilde{f}: \widetilde{H}^{3} \rightarrow \widetilde{H}^{3}$ and the limit of the fuzzy retractions $\lim _{m \rightarrow \infty} \widetilde{r}_{m}: \widetilde{H}^{3} \rightarrow \widetilde{H}^{2}$ is discussed from the following commutative diagram.

Proof. Let the fuzzy folding is $\widetilde{f}_{1}: \widetilde{H}^{3} \rightarrow \widetilde{H}^{3}$, the limit of the fuzzy retractions of $\widetilde{H}^{3}$ and $\widetilde{f}_{1}\left(\widetilde{H}^{3}\right)$ are $\lim _{m \rightarrow \infty} \widetilde{r}_{m}: \widetilde{H}^{3}$ $\rightarrow \widetilde{H}^{2}$ and $\lim _{m+1} \rightarrow \infty \widetilde{r}_{m+1}: \widetilde{f}_{1}\left(\widetilde{H}^{3}\right) \rightarrow \widetilde{H}^{2}$, and $\widetilde{f}_{2}:$ $\left(\lim _{m \rightarrow \infty} \widetilde{r}_{m}\left(\widetilde{H}^{3}\right)\right) \rightarrow \widetilde{H}^{2}$. Then, the following diagram is commutative:

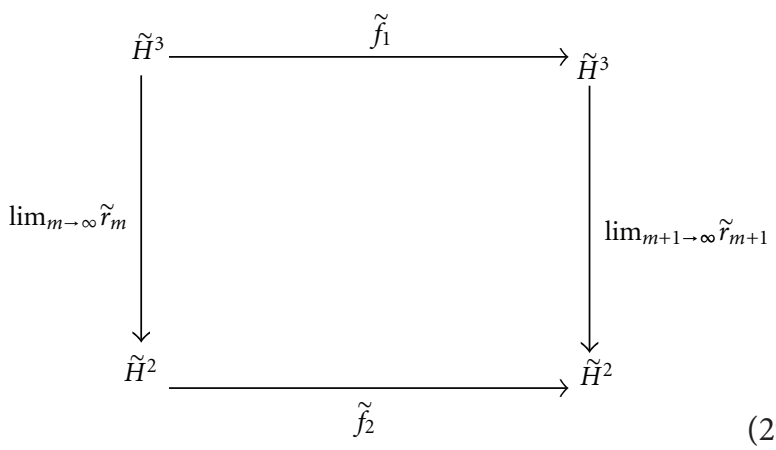

that is, $\lim _{m+1} \rightarrow \infty \widetilde{r}_{m+1} \circ \widetilde{f}_{1}\left(\widetilde{H}^{3}\right)=\tilde{f}_{2} \circ \lim _{m \rightarrow \infty} \widetilde{r}_{m}\left(\widetilde{H}^{3}\right)$, and the corresponding relations between the two chains of fuzzy folding and the limit of fuzzy retraction are given by

$$
\begin{aligned}
& \overline{\lim _{m+1 \rightarrow \infty} r_{m+1}} \circ \overline{\tilde{f}_{1}}=\overline{\tilde{f}_{2}} \circ \overline{\widetilde{\lim }_{m \rightarrow \infty} r_{m}}, \\
& \underline{\lim _{m \rightarrow \infty} r_{m+1}} \circ \tilde{f}_{1}=\tilde{f}_{2} \circ \widetilde{\varlimsup_{m \rightarrow \infty} r_{m}} .
\end{aligned}
$$

Theorem 8. Let the fuzzy retraction of $\widetilde{H}^{3}$ is $\widetilde{r}: \widetilde{H}^{3} \rightarrow$ $\widetilde{H}^{2}, \widetilde{H}^{2} \subset \widetilde{H}^{3}$, and the fuzzy folding of $\widetilde{H}^{3}$ is $\widetilde{f}: \widetilde{H}^{3} \rightarrow \widetilde{H}^{3}$, then

(i) $\tilde{f}_{2} \circ \widetilde{r}_{1}\left(\widetilde{H}^{3}\right)=\widetilde{r}_{2} \circ \tilde{f}_{1}\left(\widetilde{H}^{3}\right)$

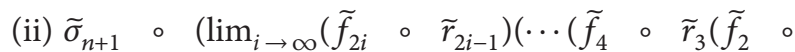
$\left.\left.\left.\left.\widetilde{r}_{1}\left(\widetilde{H}^{3}\right)\right)\right) \cdots\right)\right)=\left(\lim _{i \rightarrow \infty}\left(\widetilde{r}_{2 i} \circ \widetilde{f}_{2 i-1}\right)\left(\cdots\left(\widetilde{r}_{4} \circ \widetilde{f}_{3}\left(\widetilde{r}_{2} \circ\right.\right.\right.\right.$ $\left.\left.\left.\tilde{f}_{1}\left(\widetilde{H}^{3}\right)\right)\right) \cdots\right)$ ○) $\widetilde{\sigma}_{1}$.

Proof. (i) Let the fuzzy retraction of the fuzzy hyperboloid in $\widetilde{H}^{3} \subset \widetilde{W}^{4}$ model be $\widetilde{r}_{1}: \widetilde{H}^{3} \rightarrow \widetilde{H}^{2} ; \widetilde{f}_{1}: \widetilde{H}^{3} \rightarrow \widetilde{H}^{3}$, the fuzzy retraction of $\widetilde{f}_{1}\left(\widetilde{H}^{3}\right)$ is folding of $\widetilde{r}_{1}\left(\widetilde{H}^{3}\right)$ is $\widetilde{f}_{2}$ : $\widetilde{r}_{1}\left(\widetilde{H}^{3}\right) \rightarrow \widetilde{r}_{2}: \widetilde{f}_{1}\left(\widetilde{H}^{3}\right) \rightarrow \widetilde{H}^{2}$, and the fuzzy $\widetilde{H}^{2}$. Then $\widetilde{f}_{2}$ 。 $\widetilde{r}_{1}\left(\widetilde{H}^{3}\right)=\widetilde{r}_{2} \circ \tilde{f}_{1}\left(\widetilde{H}^{3}\right)$.

(ii) Let $\tilde{f}_{2 i} \circ \widetilde{r}_{2 i-1}$ and $\widetilde{r}_{2 i} \circ \tilde{f}_{2 i-1}$ are the compositions between the fuzzy retractions and the fuzzy foldings of $\widetilde{H}^{3}$ into itself. Also, $\widetilde{\sigma_{l}}$ are the homeomorphisms. Then

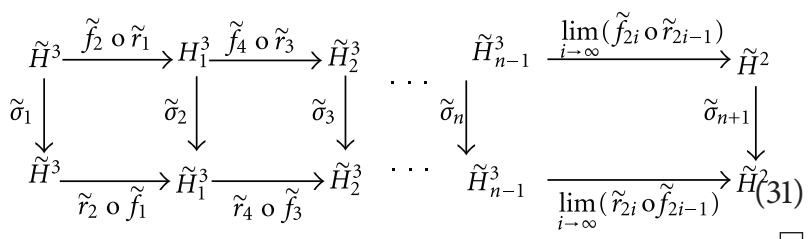

Theorem 9. Given the fuzzy deformation retract of $\widetilde{H}^{3} \subset \widetilde{W}^{4}$ model is $\widetilde{D}: \widetilde{H}^{3} \times I \rightarrow \widetilde{H}^{3}$; the limit of the fuzzy folding of $\widetilde{H}^{3} \times I$ is $\lim _{m \rightarrow \infty} \widetilde{f}_{m}: \widetilde{H}^{3} \times I \rightarrow \widetilde{H}^{2} \times I$. Then, the following diagram is commutative.

Proof. Let the limit of the fuzzy folding of $\left(\widetilde{H}^{3} \times I\right)$ is $\lim _{m \rightarrow \infty} \widetilde{f}_{m}: \widetilde{H}^{3} \times I \rightarrow \widetilde{H}^{2} \times I$, the fuzzy deformation retract of $\widetilde{H}^{3} \subset \widetilde{W}^{4}$ onto $\widetilde{H}^{2}$ is $\widetilde{D}_{1}\left(\widetilde{H}^{3} \times I\right)=\widetilde{H}^{2}$, the limit of the fuzzy folding of $\widetilde{D}_{1}\left(\widetilde{H}^{3} \times I\right)$ is $\lim _{m+1 \rightarrow \infty} \widetilde{f}_{m+1}: \widetilde{D}_{1}\left(\widetilde{H}^{3} \times I\right) \rightarrow$ $\widetilde{H}^{1}$, and the fuzzy deformation retract of $\lim _{m \rightarrow \infty} \widetilde{f}_{m}\left(\widetilde{H}^{3} \times I\right)$ onto $\widetilde{H}^{1}$ is $\widetilde{D}_{2}\left(\lim _{m \rightarrow \infty} \widetilde{f}_{m}\left(\widetilde{H}^{3} \times I\right)\right)=\widetilde{H}^{1}$. Hence

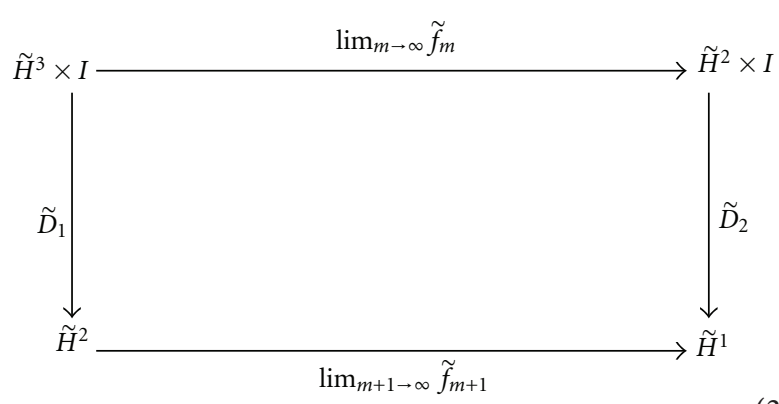

that is, $\widetilde{D}_{2} \circ \lim _{m \rightarrow \infty} \widetilde{f}_{m}\left(\widetilde{H}^{3} \times I\right)=\lim _{m+1 \rightarrow \infty} \widetilde{f}_{m+1} \circ \widetilde{D}_{1}\left(\widetilde{H}^{3} \times\right.$ I) 
Theorem 10. The composition of fuzzy deformation retracts of fuzzy hyperboloid $\widetilde{H}^{3} \subset \widetilde{W}^{4}$ model is a minimal retraction.

Proof. Now consider the following fuzzy continuous map $\tilde{\eta}$ : $\widetilde{H}^{3} \times[0,1] \rightarrow \widetilde{H}^{3}$, such that $\widetilde{\eta}(\widetilde{x}, \widetilde{s})=\widetilde{\beta}(\widetilde{x},(\widetilde{s} /(1-\widetilde{s})))$, then it is easy to see that

$$
\begin{gathered}
\tilde{\eta}(\tilde{x}, 0)=\tilde{\beta}(\tilde{x}, 0)=0, \\
\tilde{\eta}(\tilde{x}, 1)=\lim _{s \rightarrow 1} \tilde{\beta}\left(\tilde{x}, \frac{\widetilde{s}}{1-\widetilde{s}}\right)=\widetilde{S}_{1}^{2} \subset \widetilde{H}^{3}, \\
\tilde{\eta}(\tilde{y}, s)=\tilde{\beta}\left(\tilde{y}, \frac{\widetilde{s}}{1-\widetilde{s}}\right)=\widetilde{S}^{1} \subset \widetilde{S}_{1}^{2} .
\end{gathered}
$$

The fuzzy deformation retract of the fuzzy circle $\widetilde{S}^{1} \subset \widetilde{S}_{1}^{2}$ onto minimal fuzzy retraction $((0,1), \mu)$ is given in polar coordinates by

$$
\begin{aligned}
& \tilde{\mu}\left(r(\mu) e^{i \theta(\mu)}\right) \\
& = \begin{cases}\widetilde{r} e^{i(1-r(\mu)) \theta(\mu)}, & |\theta(\mu)| \leq \frac{\pi}{2}, \\
\widetilde{r} e^{i\{\theta(\mu)-(\pi-\theta(\mu)) r(\mu)\}}, & \frac{\pi}{2} \leq \theta(\mu) \leq \pi, \\
\widetilde{r} e^{i\{\theta(\mu)+(\pi+\theta(\mu)) r(\mu)\}}, & -\pi \leq \theta(\mu) \leq-\frac{\pi}{2},\end{cases}
\end{aligned}
$$

that is, $\tilde{\mu} \circ \widetilde{\eta}$ is a fuzzy minimal retraction.

Theorem 11. Let $\widetilde{H}^{3} \subset \quad \widetilde{W}^{4}$ be a fuzzy hyperboloid in $\widetilde{W}^{4}$ model which is homeomorphic to $\left\{\widetilde{D}^{2}-\widetilde{\beta}\right\} \subset \widetilde{R}^{3}, \widetilde{P}_{1}: \widetilde{H}^{3} \rightarrow$ $\left\{\widetilde{D}^{2}-\widetilde{\beta}\right\}$, the fuzzy retraction $\widetilde{r}_{1}: \widetilde{H}^{3} \rightarrow \widetilde{H}^{2}$, and the limit fuzzy folding of $\widetilde{D}^{2}$ is $\lim _{n \rightarrow \infty} \widetilde{f}_{n}:\left\{\widetilde{D}^{2}-\widetilde{\beta}\right\} \rightarrow \widetilde{D}^{1}$. Then there are induced fuzzy retraction, limit fuzzy folding, and homeomorphism map such that the following diagram is commutative:

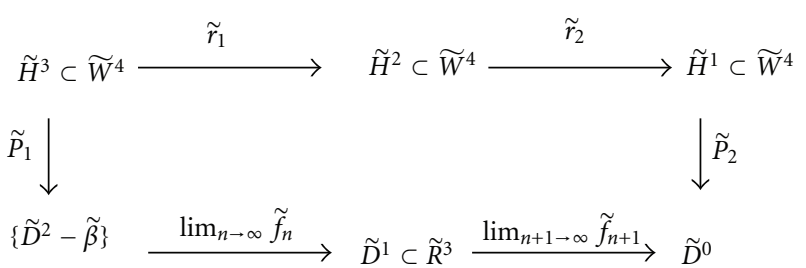

Proof. Let the homeomorphism map, $\widetilde{P}_{1}: \widetilde{H}^{3} \rightarrow\left\{\widetilde{D}^{2}-\widetilde{\beta}\right\}$ and $\widetilde{r}_{1}: \widetilde{H}^{3} \rightarrow \widetilde{H}^{2}$, also, $\lim _{n \rightarrow \infty} \widetilde{f}_{n}:\left\{\widetilde{D}^{2}-\widetilde{\beta}\right\} \rightarrow \widetilde{D}^{1}$, the fuzzy retraction of $\widetilde{r}_{1}\left(\widetilde{H}^{3}\right)$ be $\widetilde{r}_{2}: \widetilde{H}^{2} \rightarrow \widetilde{H}^{1}$; the limit fuzzy folding of $\lim _{n \rightarrow \infty} \widetilde{f}_{n}\left(\left\{\widetilde{D}^{2}-\widetilde{\beta}\right\}\right)$ is given by $\lim _{n+1} \rightarrow \infty \quad \widetilde{f}_{n+1}: \widetilde{D}^{1} \rightarrow \widetilde{D}^{0}$, and $\widetilde{P}_{2}: \widetilde{r}_{2}\left(\widetilde{r}_{1}\left(\widetilde{H}^{3}\right)\right) \rightarrow \widetilde{D}^{0}$. This proves that the diagram is commutative.

Theorem 12. If the limit fuzzy folding of the fuzzy hyperboloid $\widetilde{H}^{3} \subset \widetilde{W}^{4}$ is $\lim _{n \rightarrow \infty} \widetilde{f}_{n}: \widetilde{H}^{3} \rightarrow \widetilde{H}^{2}$, the fuzzy retraction of $\widetilde{H}^{3} \subset \widetilde{W}^{4}$ is $\widetilde{r}_{1}: \widetilde{H}^{3} \rightarrow \widetilde{H}^{2}$ and the homeomorphism map of $\widetilde{H}^{2} \subset \widetilde{W}^{4}$ is $\widetilde{P}_{1}: \widetilde{H}^{2} \rightarrow \widetilde{D}^{1}$. Then there are induced limit fuzzy retractions, limit fuzzy folding, and homeomorphism map such that the following diagram is commutative:

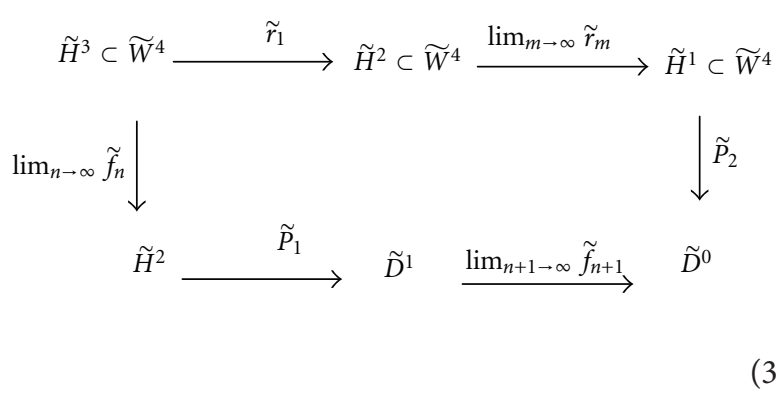

Proof. Consider the limit fuzzy folding of the fuzzy hyperboloid $\widetilde{H}^{3} \subset \widetilde{W}^{4}$ is $\lim _{n \rightarrow \infty} \widetilde{f}_{n}: \widetilde{H}^{3} \rightarrow \widetilde{H}^{2}$, the fuzzy retraction of $\widetilde{H}^{3} \subset \widetilde{W}^{4}$ is $\widetilde{r}_{1}: \widetilde{H}^{3} \rightarrow \widetilde{H}^{2}$, and the homeomorphism map of $\widetilde{H}^{2} \subset \widetilde{W}^{4}$ is $\widetilde{P}_{1}: \widetilde{H}^{2} \rightarrow \widetilde{D}^{1}$, the limit fuzzy retraction of $\widetilde{r}_{1}\left(\widetilde{H}^{3}\right)$ is $\lim _{m \rightarrow \infty} \widetilde{r}_{m}: \widetilde{H}^{2} \rightarrow \widetilde{H}^{1}$, the limit fuzzy folding of $\widetilde{P}_{1}\left(\widetilde{H}^{2}\right)$ is $\lim _{n+1 \rightarrow \infty} \widetilde{f}_{n+1}: \widetilde{D}^{1} \rightarrow$ $\widetilde{D}^{0}$, and $\widetilde{P}_{2}: \lim _{m \rightarrow \infty} \widetilde{r}_{m}\left(\widetilde{r}_{1}\left(\widetilde{H}^{3}\right)\right) \rightarrow \widetilde{D}^{0}$. This proves that the diagram is commutative.

Theorem 13. Let $\widetilde{D \cdot R}: \widetilde{H}^{3} \times I \rightarrow \widetilde{H}^{3}$ and $\widetilde{r}_{1}: \widetilde{H}^{3} \times I \rightarrow$ $\widetilde{H}^{2}$ be a fuzzy retraction, and also $\widetilde{f}: \widetilde{H}^{3} \times I \rightarrow \widetilde{H}^{3} \times$ I a fuzzy folding. Then, there is induced limit fuzzy folding $\lim _{n \rightarrow \infty} \widetilde{f}_{n}: \widetilde{H}^{3} \rightarrow \widetilde{H}^{2}$ such that the following diagram is commutative:

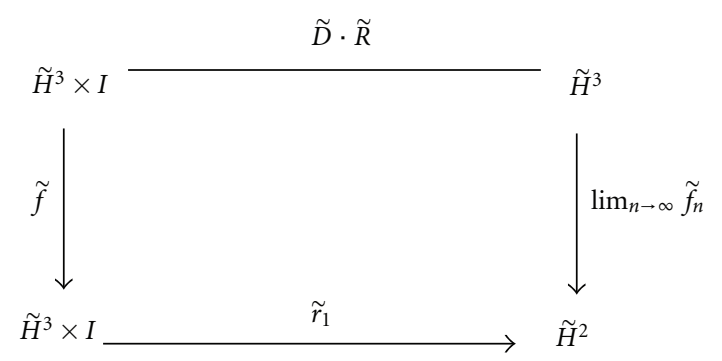

Proof. Let $\widetilde{D \cdot R}: \widetilde{H}^{3} \times I \rightarrow \widetilde{H}^{3}$, and the fuzzy folding $\tilde{f}$ : $\widetilde{H}^{3} \times I \rightarrow \widetilde{H}^{3} \times I$, also $\widetilde{r}_{1}: \widetilde{H}^{3} \times I \rightarrow \widetilde{H}^{2}$, and the limit fuzzy folding $\lim _{\mathrm{n} \rightarrow \infty} \widetilde{f}_{n}: \widetilde{H}^{3} \rightarrow \widetilde{H}^{2}$. This proves that the diagram is commutative.

Theorem 14. If the fuzzy retraction of the fuzzy hyperboloid $\widetilde{H}^{3} \subset \widetilde{W}^{4}$ is $\widetilde{r}: \widetilde{H}^{3} \times I \rightarrow \widetilde{H}^{2} \times I$, the fuzzy deformation retract of $\widetilde{H}^{3} \times I \subset \widetilde{W}^{4}$ is $\widetilde{D}_{1}: \widetilde{H}^{3} \times I \rightarrow \widetilde{H}^{3}$, and the fuzzy deformation retract of $\widetilde{H}^{2}$ is $\widetilde{D}_{2}: \widetilde{H}^{2} \times I \rightarrow \widetilde{H}^{2}$. Then there are induced end limit fuzzy retractions and fuzzy folding such that the following diagram is commutative.

Proof. Let $\widetilde{r}_{1}: \widetilde{H}^{3} \times I \rightarrow \widetilde{H}^{2} \times I$ and $\widetilde{D}_{1}: \widetilde{H}^{3} \times I \rightarrow$ $\widetilde{H}^{3}$ also, $\widetilde{D}_{2}: \widetilde{r}_{1}\left(\widetilde{H}^{3} \times I\right) \rightarrow \widetilde{H}^{2}$; the end limits fuzzy retractions of $\widetilde{D}_{1}\left(\widetilde{H}^{3} \times I\right)$ are end $\lim \widetilde{r}_{1}: \widetilde{D}_{1}\left(\widetilde{H}^{3} \times I\right) \rightarrow 0$; the end limits fuzzy retractions of $\widetilde{D}_{2}\left(\widetilde{H}^{2} \times I\right)$ are end $\lim r_{2}$ : 
$\left.\widetilde{D}_{2}\left(\widetilde{H}^{2} \times I\right)\right) \rightarrow 0$, and $\widetilde{f}:$ end $\lim \widetilde{r}_{1}\left(\widetilde{D}_{1}\left(\widetilde{H}^{3} \times I\right) \rightarrow 0\right.$, then the following diagram is commutative,

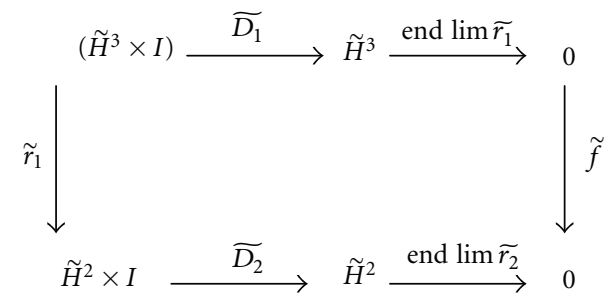

that is, $\widetilde{f} \circ\left(\right.$ end $\left.\lim \widetilde{r}_{1} \circ \widetilde{D}_{1}\left(\widetilde{H}^{3} \times I\right)\right)=$ end $\lim \widetilde{r}_{2} \circ\left(\widetilde{D}_{2} \circ \widetilde{r}_{1}\right)$.

Also, the corresponding relation is described by the two induced chains, that is,

$$
\begin{aligned}
& \overline{\widetilde{f}} \circ \overline{\left(\text { end } \lim \widetilde{r}_{1} \circ \widetilde{D}_{1}\left(\widetilde{H}^{3} \times I\right)\right)}=\overline{\text { end } \lim \widetilde{r}_{2}} \overline{\left(\widetilde{\left.D_{2} \circ \widetilde{r}_{1}\right)}\right.}, \\
& \widetilde{f} \circ \underline{\left(\text { end } \lim \widetilde{r}_{1} \circ \widetilde{D}_{1}\left(\widetilde{H}^{3} \times I\right)\right)}=\text { end } \lim \widetilde{r}_{2} \circ \underline{\left(\widetilde{D}_{2} \circ \widetilde{r}_{1}\right) .}
\end{aligned}
$$

Theorem 15. Let $\widetilde{H}^{3} \subset \widetilde{W}^{4}$ be the fuzzy hyperboloid, then the relations between the fuzzy deformation retract and the limit fuzzy folding is discussed from the following commutative diagram.

Proof. Let the fuzzy deformation retract $\widetilde{D}_{1}: \widetilde{H}^{3} \times I \rightarrow$ $\widetilde{H}^{3}$; the limit fuzzy folding of $\widetilde{H}^{3}$ and $\widetilde{D}_{1}\left(\widetilde{H}^{3} \times I\right)$ is $\lim _{m \rightarrow \infty} \tilde{f}_{m}: \widetilde{H}^{3} \times I \rightarrow \widetilde{H}^{2} \times I$ and $\lim _{m+1 \rightarrow \infty} \widetilde{f}_{m+1}:$ $\widetilde{D}_{1}\left(\widetilde{H}^{3} \times I\right) \rightarrow \widetilde{H}^{2}$, and $\widetilde{D}_{2}:\left(\lim _{m \rightarrow \infty} \widetilde{f}_{m}\left(\widetilde{H}^{3} \times I\right)\right) \rightarrow$ $\widetilde{H}^{2}$. Then, we have the following diagram,

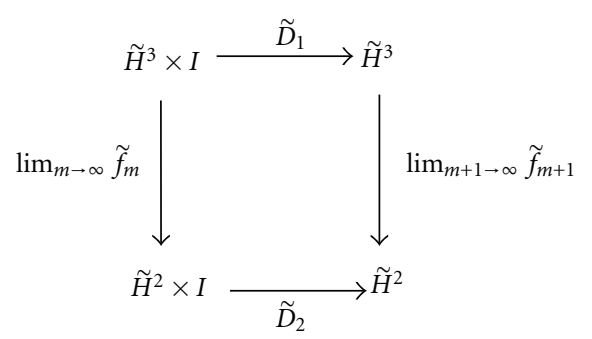

that is, $\lim _{m+1 \rightarrow \infty} \widetilde{f}_{m+1} \circ \widetilde{D}_{1}\left(\widetilde{H}^{3} \times I\right)=\widetilde{D}_{2} \circ \lim _{m \rightarrow \infty} \widetilde{f}_{m}\left(\widetilde{H}^{3} \times\right.$ I).

Also, the corresponding relations are described by the two induced chains, that is,

$$
\begin{aligned}
& \overline{\lim _{m+1 \rightarrow \infty} f_{m+1}} \circ \overline{\widetilde{D}_{1}}=\overline{\widetilde{D}_{2}} \circ \overline{\lim _{m \rightarrow \infty} f_{m}},
\end{aligned}
$$

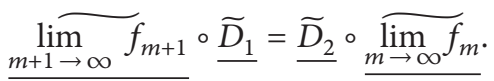

\section{Conclusion}

In the present paper, we obtain and study new types of fuzzy retractions of $\widetilde{W}^{4}$ model. Also, we deduced new types of fuzzy deformation retract of $\widetilde{W}^{4}$ model. The relations between the fuzzy folding and the fuzzy deformation retracts of $\widetilde{W}^{4}$ model is obtained. New types of minimal fuzzy retraction of $\widetilde{W}^{4}$ model is also presented. New types of homotopy maps are described. The isometric and topological fuzzy folding in each case and the relation between the fuzzy deformation retract after and before fuzzy folding have been obtained. Types of conditional fuzzy folding of $\widetilde{W}^{4}$ model are described.

\section{References}

[1] B. Schmeikal, Primordial Space-The Metric Case, Nova Science, New York, NY, USA, 2010.

[2] F. Catoni, D. Boccaletti, R. Cannata, V. Catoni, and P. Zampetti, Geometry of Minkowski Space-Time, Birkhauser, Boston, Mass, USA, 2011.

[3] F. Catoni, D. Boccaletti, R. Cannata, V. Catoni, E. Nichelatti, and P. Zampetti, The Mathematics of Minkowski Space-Time, Birkhauser, Boston, Mass, USA, 2008.

[4] J. B. Hartle, Gravity, An Introduction to Einstein's General Relativity, Addison-Wesley, New York, NY, USA, 2003.

[5] J. B. Griffiths and J. Podolsky, Exact Space-Times in Einstein's General Relativity, Cambridge University Press, Cambridge, UK, 2009.

[6] N. Straumann, General Relativity with Application to Astrophysics, Springer, New York, NY, USA, 2004.

[7] A. E. El-Ahmady, "Folding of fuzzy hypertori and their retractions," Proceedings of the Mathematical and Physical Society of Egypt, vol. 85, no. 1, pp. 1-10, 2007.

[8] A. E. El-Ahmady, "Limits of fuzzy retractions of fuzzy hyperspheres and their foldings," Tamkang Journal of Mathematics, vol. 37, no. 1, pp. 47-55, 2006.

[9] G. L. Naber, Topology, Geometry and Gauge Fields: Foundations, Texts in Applied Mathematics 25, Springer, New York, NY, USA, 2nd edition, 2011.

[10] G. L. Naber, Topology, Geometry and Gauge Fields: Interactions, Applied Mathematics Sciences, Springer, New York, NY, USA, 2nd edition, 2011.

[11] M. Reid and B. Szendroi, Topology and Geometry, Cambridge University Press, Cambridge, UK, 2005.

[12] A. E. El-Ahmady, "The variation of the density functions on chaotic spheres in chaotic space-like Minkowski space time," Chaos, Solitons and Fractals, vol. 31, no. 5, pp. 1272-1278, 2007.

[13] A. E. El-Ahmady and A. El-Araby, "On fuzzy spheres in fuzzy Minkowski space," Nuovo Cimento della Societa Italiana di Fisica B, vol. 125, no. 10, pp. 1153-1160, 2010.

[14] A. E. El-Ahmady, "Fuzzy Lobachevskian space and its folding," The Journal of Fuzzy Mathematics, vol. 12, no. 2, pp. 609-614, 2004.

[15] A. E. El-Ahmady, "Fuzzy folding of fuzzy horocycle," Circolo Matematico Di Palermo Serie II, vol. 53, no. 3, pp. 443-450, 2004.

[16] A. P. Balachandran, S. Kurkcuoglu, and S. Vaidya, Lectures on Fuzzy and Fuzzy Susy Physics, World Scientific Publishing, New York, NY, USA, 2007.

[17] L. A. Zadeh, Fuzzy Sets and Their Application to Congnitive and Decision, Academic Press, New York, NY, USA, 1975.

[18] N. Palaniappan, Fuzzy Topology, Alpha Science International, London, UK, 2005. 

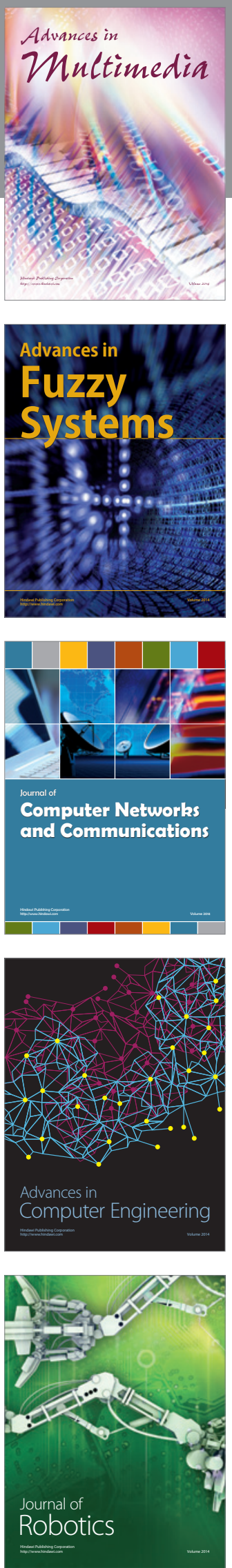

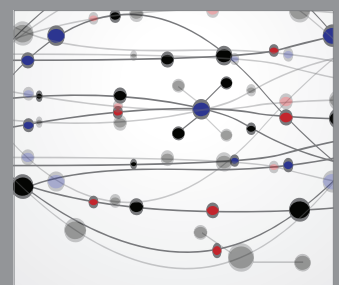

The Scientific World Journal
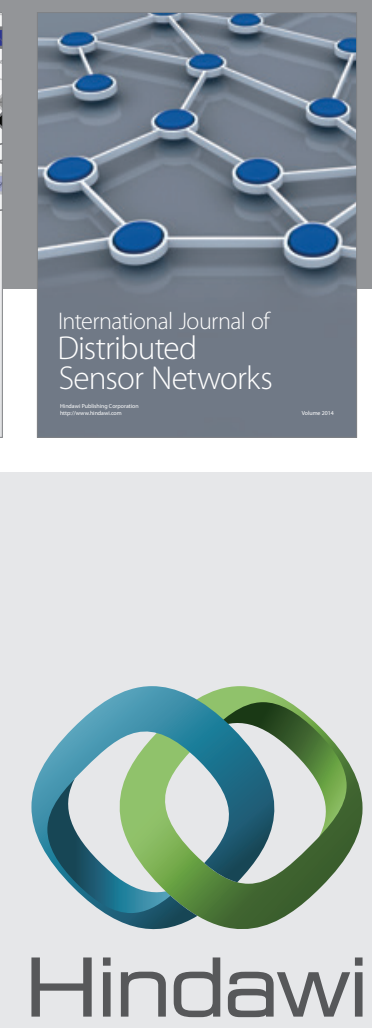

Submit your manuscripts at

http://www.hindawi.com
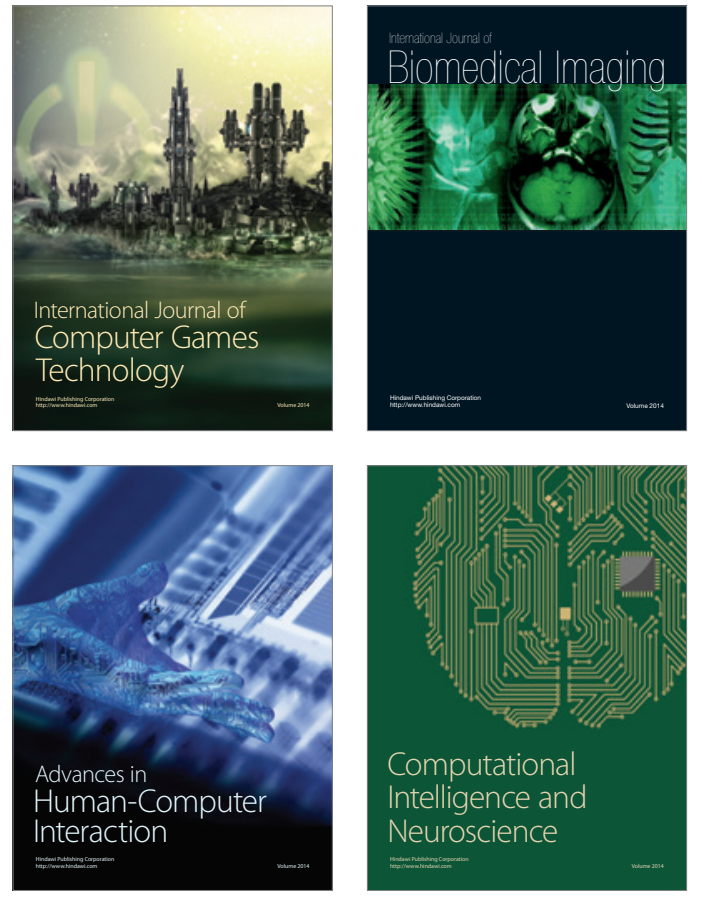
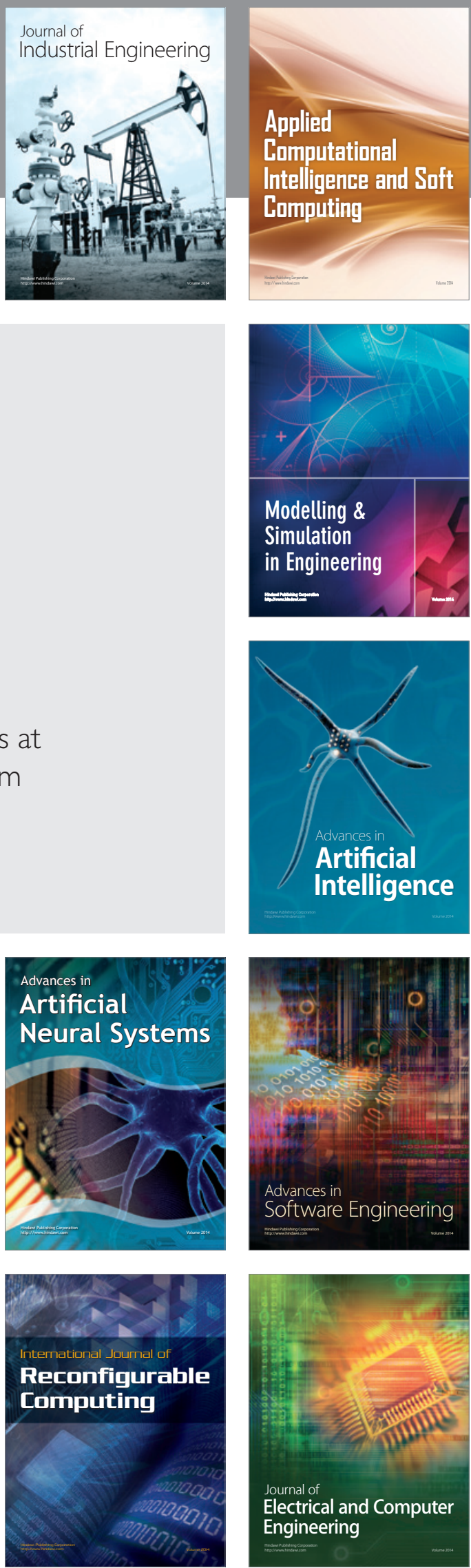